\title{
BINARY SUPERSOLVABLE MATROIDS AND MODULAR CONSTRUCTIONS
}

\author{
GÜNTER M. ZIEGLER
}

(Communicated by Thomas H. Brylawski)

\begin{abstract}
Let $\mathscr{M}$ be the class of binary matroids without a Fano plane as a submatroid. We show that every supersolvable matroid in $\mathscr{M}$ is graphic, corresponding to a chordal graph. Then we characterize the case that the modular join of two matroids is supersolvable. This is used to study modular flats and modular joins of binary supersolvable matroids. We decompose supersolvable matroids in $\mathscr{M}$ as modular joins with respect to hyperplanes. For such matroids every modular flat is contained in a maximal chain of modular flats, and thus modular joins are again supersolvable.
\end{abstract}

\section{INTRODUCTION}

Supersolvable matroids were introduced and studied by R. P. Stanley [17] in 1972. He found that a graphic matroid is supersolvable if and only if the corresponding graph is chordal (triangulated).

In this paper we extend this result to the class $\mathscr{M}$ of binary matroids that do not contain a Fano plane as a submatroid (Theorem 2.7): every supersolvable binary matroid without a Fano submatroid is graphic (corresponding to a chordal graph).

We then study modular flats and modular joins of supersolvable matroids. Our main result is a characterization of the case that the modular join of two matroids is supersolvable (Theorem 3.4). We also show that the class of geometric lattices that arise from supersolvable ones by modular joins is closed under taking intervals (Theorem 3.9).

Finally the special structure in the case of supersolvable matroids in $\mathscr{M}$ is explored. Here every modular flat extends to an $M$-chain, such that modular joins of supersolvable matroids in $\mathscr{M}$ are again supersolvable (Theorem 4.7).

It is assumed that the reader is familiar with basic matroid theory, as developed in [18-20]. The basic references on modularity are Stanley [16] for modular flats, [17] for supersolvable matroids, and Brylawski [5] for modular constructions. Definitions and the basic properties needed for this paper will

Received by the editors December 27, 1988.

1980 Mathematics Subject Classification (1985 Revision). Primary 05B35; Seconary 06C10. 
be reviewed in $\S 2$. Chordal (triangulated) graphs are treated extensively in [9, Chapter 4].

In the following, a matroid $M$ on the ground set $E$ will be denoted by $M$ or $M(E)$. Matroids will usually be represented by their lattices $L$ of flats (closed sets), where $L \subseteq 2^{E}$ is ordered by inclusion. All matroids are finite. Without loss of generality the matroids considered can be assumed to be simple (combinatorial geometries), so that the ground set $E$ is identified with the set of atoms of $L$.

A flat $X \in L$ is often identified with the corresponding matroid restriction $M \mid X$, or equivalently, with the corresponding interval $[\hat{0}, X]$ of $L$. Similarly, the contraction $M / X$ by a flat $X$ is visualized by the interval $[X, \hat{1}]$ of $L$ (where $\hat{0}=\bar{\varnothing}$ and $\hat{\mathrm{i}}=E$ ).

Our language will mostly be geometric, talking about "points and hyperplanes" rather than about "atoms and coatoms" or about "flats of rank 1 and $r-1$ " etc. The reader should have no difficulty in translating to his favorite language.

\section{BINARY SUPERSOLVABLE MATROIDS}

We start by reviewing the basic properties of modular flats in geometric lattices.

Definition 2.1 [16]. A flat $X \in L$ is modular if and only if any of the following equivalent conditions holds:

(i) $r(X)+r(Y)=r(X \wedge Y)+r(X \vee Y)$ for every $Y \in L$.

(ii) Every complement of $X$ has rank $r(L)-r(X)$.

(iii) For $Y \leq Z$ in $L, Y \vee(X \wedge Z)=(Y \vee X) \wedge Z$.

(iii') For $Y \in L$ and $X^{\prime} \leq X, X^{\prime} \vee(Y \wedge X)=\left(X^{\prime} \vee Y\right) \wedge X$.

The following result describes the main mechanisms that lead to the construction of new modular elements.

Proposition 2.2. Let $L$ be a geometric lattice, $X \in L$ a modular flat. Then

(1a) If $Y \leq X$, where $Y$ is modular in $[0, X]$, then $Y$ is modular in $L$.

(1b) If $Y \geq X$, where $Y$ is a separator (distributive) in $[X, \hat{1}]$, then $Y$ is modular in $L$.

(2) If $Y$ and $Z$ are flats in $L$ with $Y \leq Z$, then

$$
\bar{X}:=Y \vee(X \wedge Z)=(Y \vee X) \wedge Z
$$

is a modular flat of $[Y, Z]$.

Proof. (1a) is implicit in [16], explicit in [5, Proposition 3.5]. (1b) is implied by [5, Proposition 3.19]. (2) is a combination of [16, Lemma 2] with [5, Corollary 3.19]. It can also be derived directly from Definition 2.1(i) or (iii).

See [5, Theorem 3.3] for a wealth of characterizations of modular flats. The basic properties are collected in the following. 
Proposition 2.3 [16, 5]. Let $M$ be a matroid.

(1) $\hat{0}, E$ and the atoms of $L$ are always modular flats. A hyperplane $H$ of $M$ is modular if and only if it meets every line, that is, if for $p^{\prime}$, $p^{\prime \prime} \in E-H$ with $\overline{p^{\prime}} \neq \overline{p^{\prime \prime}}$ there is a $p \in H$ that lies on the line $\overline{\left\{p, p^{\prime}\right\}}$ determined by $p^{\prime}$ and $p^{\prime \prime}$.

(2) The meet (intersection) of modular flats is always modular.

(3) Modular flats in connected matroids are always connected. A flat is modular in $M$ if and only if the intersection with every connected component (minimal separator) of $M$ is modular.

(4) If $X$ is modular in $M$, then it is also modular in every restriction $M \mid S$ to a subset $S \subseteq E$ that contains $X$.

Proof. Some of this is implicit in [16]. Explicit statements and proofs can be found in [5]: (1) is [5, Corollary 3.4] (this is trivial from our definition); (2) is [5, Proposition 3.6] (this follows directly from our Proposition 2.2); (3) is [5, Corollary 3.16]; and (4) is [5, Proposition 3.8].

Modular flats are important structures in a matroid. For example, they induce a factorization of the characteristic polynomial (Stanley's "Modular Factorization Theorem," [16]), and allow various constructions, see $\S 3$.

This is exploited in the nice special case of supersolvable matroids, for which the characteristic polynomial factors completely:

Definition 2.4 [17]. A matroid $M$ is supersolvable if its lattice $L$ contains a maximal chain of modular elements ( $M$-chain). Equivalently, a matroid is supersolvable if it either has rank at most 2 , or rank $r>2$ and a modular hyperplane $H$ such that the restriction $M \mid H$ is supersolvable of rank $r-1$.

Theorem 2.5 [17]. (1) Let $M$ be a simple supersolvable matroid, let $\varnothing=X_{0} \lessdot$ $\cdots<X_{r}=E$ be an $M$-chain and $e_{i}=\left|X_{i}\right|-\left|X_{i-1}\right|$ for $1 \leq i \leq r$. Then the characteristic polynomial of $L$ is

$$
\chi(t)=\prod_{i=1}^{r}\left(t-e_{i}\right) .
$$

(2) Every restriction of a supersolvable matroid to one of its flats is again supersolvable. In fact every interval in a supersolvable geometric lattice is supersolvable.

Trivially, every modular geometric lattice is supersolvable. Thus the full projective geometry $\mathrm{PG}(n, q)$ over a finite field is always supersolvable.

A second class of supersolvable geometries is given by

Proposition 2.6 [17]. A graphic matroid $M(G)$ is supersolvable if and only if $G$ is chordal.

(The "only if" part is clear from Theorem 2.5(2). The "if" direction relies on the existence of a simplicial vertex in every chordal graph, see Lemma 4.5.) 
Now we will see that every regular (unimodular) supersolvable matroid is graphic. The Fano plane $F_{7}=\operatorname{PG}(2,2)$ shows that this does not generalize to binary matroids, and the $(q+1)$-point line $U_{2, q+1}=\operatorname{PG}(1, q)$ for $q>2$ is a counterexample over other fields.

However, one can consider the class $\mathscr{M}$ of binary matroids that does not contain a Fano plane as a submatroid. Note that in a simple binary matroid, every Fano submatroid is a flat. For matroids in $\mathscr{M}$ we formulate the main result of this note; this in particular covers the class of all regular matroids, and thus the case of all graphic and cographic matroids.

For our purposes we describe a simple binary matroid $M$ of rank $r$ as graphic if in suitable coordinates over $\mathrm{GF}(2)$ it is a subset of $M\left(K_{r+1}\right)=$ $\left\{\mathbf{e}_{i}+\mathbf{e}_{j}: 1 \leq i<j \leq r+1\right\}$, where $\mathbf{e}_{i}$ is the $i$ th unit vector in $V=\operatorname{GF}(2)^{r+1}$.

Theorem 2.7. Let $M$ be a supersolvable binary matroid. If $M$ does not contain a Fano plane as a submatroid, then it is graphic. In other words, every supersolvable $M \in \mathscr{M}$ is graphic.

Proof. Let $M$ be a supersolvable binary matroid on $E$ of rank $r=r(M)$. We can assume that $M$ is simple.

Let $H \subseteq E$ be a modular hyperplane. $M$ will be represented in the subspace $V$ of $\mathrm{GF}(2)^{r+1}$ defined by $\sum_{i=1}^{r+1} x_{i}=0$. By induction on the rank we may assume that the restriction $M \mid H$ is graphic and represented as $H \subseteq\left\{\mathbf{e}_{i}+\mathbf{e}_{j}\right.$ : $1 \leq i<j \leq r\}$, where $\mathbf{e}_{i}$ denotes the $i$ th coordinate vector in $\operatorname{GF}(2)^{r+1}$, thus $\mathbf{e}_{i}+\mathbf{e}_{j} \subseteq\left\{\mathbf{x} \in V: x_{r+1}=0\right\}$. Now let $E-H=\left\{\mathbf{f}_{1}, \ldots, \mathbf{f}_{k}\right\} \subseteq\left\{\mathbf{x} \in V: x_{r+1}=\right.$ 1\}. Without loss of generality we may assume $\mathbf{f}_{1}=\mathbf{e}_{1}+\mathbf{e}_{r+1}$, and also (if $k \geq 2$ ) that the point in $H$ determined by $\mathbf{f}_{1}$ and $\mathbf{f}_{2}$ as $H \cap \overline{\left\{\mathbf{f}_{1}, \mathbf{f}_{2}\right\}}=\left\{\mathbf{f}_{1}+\mathbf{f}_{2}\right\}$ is $\mathbf{e}_{1}+\mathbf{e}_{2}$, after permuting coordinates. This implies $\mathbf{f}_{2}=\mathbf{e}_{2}+\mathbf{e}_{r+1}$.

There are several possibilities for $\mathbf{f}_{3}$. The conditions $\mathbf{f}_{3}+\mathbf{f}_{1} \in H$ and $\mathbf{f}_{3}+\mathbf{f}_{2} \in$ $H$ and $\mathbf{f}_{3} \notin H$ imply that either $\mathbf{f}_{3}=\mathbf{e}_{j}+\mathbf{e}_{r+1}$ for some $j$ with $3 \leq j \leq r$ (in which case we can assume $\mathbf{f}_{3}=\mathbf{e}_{3}+\mathbf{e}_{r+1}$ after a permutation of coordinates), or that $\mathbf{f}_{3}=\mathbf{e}_{1}+\mathbf{e}_{2}+\mathbf{e}_{j}+\mathbf{e}_{r+1}$ for some $3 \leq j \leq r$. by

In this case we perform an invertible linear coordinate transformation given

$$
\begin{aligned}
\mathbf{e}_{1} & \mapsto \mathbf{e}_{1} \\
\mathbf{e}_{2} & \mapsto \mathbf{e}_{2} \\
\mathbf{e}_{r+1} & \mapsto \mathbf{e}_{r+1} \\
\mathbf{e}_{k} & \mapsto \mathbf{e}_{k}+\mathbf{e}_{1}+\mathbf{e}_{2} \quad \text { for } 3 \leq k \leq r,
\end{aligned}
$$

which fixes $V$, fixes $\left\{\mathbf{e}_{i}+\mathbf{e}_{j}: 1 \leq i<j \leq r\right\}$, fixes $\mathbf{f}_{1}$ and $\mathbf{f}_{2}$, and sends $\mathbf{f}_{3}$ to $\mathbf{e}_{j}+\mathbf{e}_{r+1}$, such that after an additional permutation of coordinates we may assume $\mathbf{f}_{3}=\mathbf{e}_{3}+\mathbf{e}_{r+1}$.

But now for $\mathbf{f}_{k}$ with $k \geq 4$ we get the conditions $\mathbf{f}_{1}+\mathbf{f}_{k} \in H, \mathbf{f}_{2}+\mathbf{f}_{k} \in H$, and $\mathbf{f}_{3}+\mathbf{f}_{k} \in H$, thus either $\mathbf{f}_{k}=\mathbf{e}_{j}+\mathbf{e}_{r+1}$ for some $4 \leq j \leq r$, or $\mathbf{f}_{k}=$ $\mathbf{e}_{1}+\mathbf{e}_{2}+\mathbf{e}_{3}+\mathbf{e}_{r+1}$, but this possibility is excluded, because it creates a Fano 
plane

$\left\{\mathbf{e}_{1}+\mathbf{e}_{2}, \mathbf{e}_{1}+\mathbf{e}_{3}, \mathbf{e}_{2}+\mathbf{e}_{3}, \mathbf{e}_{1}+\mathbf{e}_{r+1}, \mathbf{e}_{2}+\mathbf{e}_{r+1}, \mathbf{e}_{3}+\mathbf{e}_{r+1}, \mathbf{e}_{1}+\mathbf{e}_{2}+\mathbf{e}_{3}+\mathbf{e}_{r+1}\right\}$.

Thus after some coordinate transformations we get $E-H \subseteq\left\{\mathbf{e}_{j}+\mathbf{e}_{r+1}: 1 \leq\right.$ $j \leq r\}$, and thus $E \subseteq\left\{\mathbf{e}_{1}+\mathbf{e}_{j}: 1 \leq i<j \leq r+1\right\}$, and thus $M$ is graphic.

We remark that the decomposition theory of [15] offers an alternative approach to Theorem 2.7. An application of the theory, using Seymour's splitter theorem, shows that a supersolvable member of $\mathscr{M}$ must be either graphic or cographic. Then remains the interesting combinatorial exercise to show that if $M^{*}(G)$ is supersolvable, then $G$ is planar.

Theorems like 2.7 in particular imply cardinality bounds:

Lemma 2.8. Let $M=M(E)$ be a simple binary matroid of rank $r$. [Thus $|E| \leq 2^{r}-1$.]

(i) If $M$ does not have a Fano minor ( $M$ is pseudo-unimodular [1]), then $|E| \leq\left(\begin{array}{c}r+1 \\ 2\end{array}\right)$. Furthermore, when equality holds, then $M$ is (isomorphic to) the graphic matroid $M\left(K_{r+1}\right)$.

(ii) If $M$ does not have a Fano submatroid $(M \in \mathscr{M})$, then $|E| \leq 3 \cdot 2^{r-2}$. Furthermore, when equality holds, then $M$ is (isomorphic to) the affine matroid $\mathrm{AG}(r, 2)$.

Proof. (i) Heller [10] and Bactawski and White [1].

(ii) This is a special case of Bose and Burton [4].

(See Murty [13] for a survey and Kung [11] for recent results on cardinality bounds of this type.)

Now if $M$ is supersolvable without a Fano submatroid, then by Theorem 2.8 it does not have a Fano minor, and thus the stronger bound of Lemma 2.8(i) applies-which is clear for graphic matroids, anyway.

Of course, it is interesting to ask for possible generalizations of Theorem 2.7. For this, one might consider concepts that generalize supersolvability (at least for binary matroids).

The standard obstruction to such attempts is the cographic matroid $M^{*}\left(K_{3,3}\right)$ : this matroid does not have a Fano submatroid and is therefore in $\mathscr{M}$. However, it is not graphic, although it is "line closed" in the sense of Halsey [9] and chordal (generalizing the definition of a chordal graph to binary matroids, as suggested by Barahona and Grötschel [2, p. 53]).

Also the characteristic polynomial of $M^{*}\left(K_{3,3}\right)$ does not factor, so that the "nice" properties of supersolvable matroids do not generalize to line closed or chordal matroids or to any other class that contains $M^{*}\left(K_{3,3}\right)$.

The best we can offer in the direction of generalization of supersolvable matroids with "good properties" is therefore the class of matroids that arise from supersolvable matroids by the modular join operation. This class will be defined and studied in the following section. In $\S 4$ we will discuss the binary case. 


\section{MOdUlaR JOINS OF SUPERSOlVABLE MATROIDS}

Definition 3.1 [5]. Let $M^{\prime}=\left(E^{\prime}, \mathscr{I}^{\prime}\right)$ and $M^{\prime \prime}=\left(E^{\prime \prime}, \mathscr{I}^{\prime \prime}\right)$ be two matroids such that $X:=E^{\prime} \cap E^{\prime \prime}$ is a modular flat in both of them. Then the modular join of $M^{\prime}$ and $M^{\prime \prime}$ is the matroid $M=P_{X}\left(M^{\prime}, M^{\prime \prime}\right)$ on $E:=E^{\prime} \cup E^{\prime \prime}$, whose closed sets are those sets $S \subseteq E$ for which $S \cap E^{\prime}$ is closed in $M^{\prime}$ and $S \cap E^{\prime \prime}$ is closed in $M^{\prime \prime}$. This defines $M$ uniquely. $M$ has rank $r(E)=$ $r\left(E^{\prime}\right)+r\left(E^{\prime \prime}\right)-r(X)$. Both $E^{\prime}$ and $E^{\prime \prime}$ are modular flats of $M$.

We say that $M$ is a modular join if it has the form $M=P_{X}\left(M\left|E^{\prime}, M\right| E^{\prime \prime}\right)$ for modular flats $E^{\prime}, E^{\prime \prime}$ of $M$ with $X=E^{\prime} \cap E^{\prime \prime}$ and $X \neq E^{\prime}, E^{\prime \prime}$ (that is, $\left.r(M)>\max \left\{r\left(E^{\prime}\right), r\left(E^{\prime \prime}\right)\right\}\right)$.

Brylawski [4] has shown that if $X \subseteq E$ is a modular flat in at least one of the geometries $M^{\prime}, M^{\prime \prime}$, then the construction in Definition 3.1 produces a matroid, which he has named the strong join or the generalized parallel connection of $M^{\prime}$ and $M^{\prime \prime}$. Lindström [13] has shown that modularity (and closedness) are required to define a symmetric join operation. We will only need this special case of the symmetric modular join construction described above. (Our terminology here is an attempt to be simple and intuitive without contradicting most of the literature.)

Proposition 3.2 [5, Corollary 6.13, Theorem 6.17]. (1) The modular join of two binary matroids is again binary.

(2) The modular join of two unimodular matroids is again unimodular.

(3) The modular join of two graphic matroids is again graphic.

To recognize modular joins, we observe the following extremely useful criterion, essentially due to Brylawski [5, Proposition 5.9]:

Proposition 3.3. Let $M$ be a matroid, and let $E^{\prime}, E^{\prime \prime} \subseteq E$ be two modular flats with $E^{\prime} \cup E^{\prime \prime}=E$. Then $M=P_{X}\left(M^{\prime}, M^{\prime \prime}\right)$ for $X=E^{\prime} \cap E^{\prime \prime}$, where $M^{\prime}=M \mid E^{\prime}$ and $M^{\prime \prime}=M \mid E^{\prime \prime}$ are the corresponding restrictions.

Proof. It suffices to see that all the closed sets of $M$ are closed in $P_{X}\left(M^{\prime}, M^{\prime \prime}\right)$ (by definition), and that $M$ and $P_{X}\left(M^{\prime}, M^{\prime \prime}\right)$ have both the same rank; namely, $r\left(E^{\prime}\right)+r\left(E^{\prime \prime}\right)-r(X)$.

From now on we will often write $P_{X}\left(E^{\prime}, E^{\prime \prime}\right)$, instead of $P_{X}\left(M\left|E^{\prime}, M\right| E^{\prime \prime}\right)$, when it is clear what matroid on $E^{\prime}$ respectively $E^{\prime \prime}$ is considered.

In this section we will characterize the case when the modular join construction produces supersolvable matroids. For this we note that if $P=P_{X}\left(M^{\prime}, M^{\prime \prime}\right)$ is supersolvable, then $M^{\prime}$ and $M^{\prime \prime}$ (being flats of $P$ ) both are supersolvable by Theorem $2.5(2)$.

From the characterization below and Example 4.3 we see that the converse is not in general true even for binary matroids, but holds for matroids in $\mathscr{M}$.

Theorem 3.4. Let $P$ be a matroid and $X$ an inclusion-minimal modular flat such that $P$ is a modular join over $X$, with $P=P_{X}\left(E^{\prime}, E^{\prime \prime}\right)$. Then $P$ is 
supersolvable if and only if $M^{\prime}$ and $M^{\prime \prime}$ are both supersolvable and $X$ belongs to an $M$-chain in both $M^{\prime}$ and $M^{\prime \prime}$.

Proof. The "if" part follows by induction on the rank from the observation that if $H^{\prime}$ is a modular hyperplane of $M^{\prime}$ that contains $X$, then $H^{\prime} \cup E^{\prime \prime}$ is a modular hyperplane of $P$ that contains $X$, and $P \mid\left(H^{\prime} \cup E^{\prime \prime}\right)=P_{X}\left(H^{\prime}, E^{\prime \prime}\right)$ by Proposition 3.3. For the converse, we first prove that every modular hyperplane $H$ of $P$ has to contain one of $E^{\prime}$ and $E^{\prime \prime}$. Assume that $p^{\prime \prime} \in E^{\prime}-H$ and $p^{\prime \prime} \in$ $E^{\prime \prime}-H$. If $p^{\prime}$ and $p^{\prime \prime}$ can be chosen such that $p^{\prime} \notin E^{\prime \prime}$ and $p^{\prime \prime} \notin E^{\prime}$, then $p^{\prime} \neq p^{\prime \prime}$, and the line $l=\overline{\left\{p^{\prime}, p^{\prime \prime}\right\}}$ has to intersect $H$ in a point $q \in E^{\prime} \cup E^{\prime \prime}$. But $q \in E^{\prime}$ would imply $l \subseteq E^{\prime}$ and thus $p^{\prime \prime} \in E^{\prime}$, and similarly $q \in E^{\prime \prime}$ leads to a contradiction. Thus we have that $E^{\prime}-E^{\prime \prime} \subseteq H$ or $E^{\prime \prime}-E^{\prime} \subseteq H$. Assume without loss of generality that the first alternative holds.

Now $X$ and $H$ are modular flats and $E^{\prime} \nsubseteq H$, so $X \nsubseteq H$. Therefore $X \cap H$ is a modular flat in $P$ that is properly contained in $X$. By Proposition 3.3, this implies $P=P_{X \cap H}\left(M^{\prime} \mid\left(H \cap E^{\prime}\right), M^{\prime \prime}\right)$, which is a contradiction to the minimality of $X$.

Now assume we have a modular hyperplane $H$ of $P$ that without loss of generality satisfies $E^{\prime} \subseteq H$. Then by Proposition 3.3 we find $P \mid H=$ $P_{X}\left(M^{\prime}, M^{\prime \prime} \mid H\right)$, and thus we are done by induction if we can verify that $X$ satisfies the minimality condition for $P \mid H$. But if on the other hand, there is a modular flat $Y \subset X$ of $H$ such that $P \mid H=P_{Y}\left(M_{1}, M_{2}\right)$, then $Y$ is a modular flat of $P$ by Proposition 2.2 (1a), and the interval $[Y, \hat{1}]$ of $P$ is a direct sum by 2.3(3). However, the corresponding separators $X_{1}, X_{2}$ of $[Y, \hat{1}]$ are modular flats of $P$ by Proposition 2.2(1b), and thus $P=P_{Y}\left(X_{1}, X_{2}\right)$ by Proposition 3.2, contradicting the minimality assumption for $X$.

Note that the "only if" part of Theorem 3.4 is not true without the minimality assumption on $X$ : for this let $M$ be a supersolvable matroid with a modular flat $X$ that is not contained in an $M$-chain (as, for example, described in Example 4.3 below). Then if $M^{\prime}$ is any supersolvable matroid, then $P=$ $P_{X}\left(M, X \oplus M^{\prime}\right)=M \oplus M^{\prime}$ is supersolvable, but $X$ is not contained in an $M$-chain of $P$.

Theorem 3.4 in particular shows that there are modular joins of supersolvable matroids (of rank at least 4 in general, of rank at least 5 in the binary case) that are not supersolvable themselves.

If $\mathscr{S}$ is the class of supersolvable geometric lattices, this produces the first combinatorially defined class $\mathscr{S}^{*}$ of geometric lattices that properly contains $\mathscr{S}$ and

(i) is closed under taking intervals, and

(ii) on which the characteristic polynomial factors.

We will see this in Theorem 3.9 after several lemmas.

Lemma 3.5. For any flat $Y$ of $P_{X}\left(M^{\prime}, M^{\prime \prime}\right)$ we have $Y \vee E^{\prime}=(Y \vee X) \cup E^{\prime}$. 
Proof. It is clear that $E^{\prime} \leq Y \vee E^{\prime}$ and $Y \vee X \leq Y \vee E^{\prime}$. Therefore we only have to show that $(Y \vee X) \cup E^{\prime}$ is closed. For this we compute

$$
\begin{aligned}
((Y \vee X) & \left.\cup E^{\prime}\right) \cap E^{\prime}=E^{\prime}, \\
\left((Y \vee X) \cup E^{\prime}\right) \cap E^{\prime \prime} & =\left((Y \vee X) \cap E^{\prime \prime}\right) \cup\left(E^{\prime} \cap E^{\prime \prime}\right) \\
& =\left((Y \vee X) \cap E^{\prime \prime}\right) \cup X \\
& =(Y \vee X) \cap E^{\prime \prime},
\end{aligned}
$$

which are closed in $E^{\prime}$ and $E^{\prime \prime}$, respectively.

Proposition 3.6. Let $[Y, Z]$ be an interval in (the lattice of flats $L_{P}$ of ) $P=$ $P_{X}\left(M^{\prime}, M^{\prime \prime}\right)$. Then $[Y, Z]$ is again the lattice of flats of a (possibly trivial) modular join; namely, of

$$
(P / Y) \mid Z=P_{\bar{X}}\left(\left(M^{\prime} / Y^{\prime}\right)\left|\overline{E^{\prime}},\left(M^{\prime \prime} / Y^{\prime \prime}\right)\right| \overline{E^{\prime \prime}}\right),
$$

where $Y^{\prime}=Y \cap E^{\prime}, Y^{\prime \prime}=Y \cap E^{\prime \prime}$, and $\bar{W}:=Y \vee(W \wedge Z)=(Y \vee W) \wedge Z$ for a modular flat $W \in L_{P}$.

Proof. $\bar{X}, \overline{E^{\prime}}$, and $\overline{E^{\prime \prime}}$ are well-defined modular flats in $[Y, Z]$ by Proposition 2.2(2). Using Lemma 3.5 , we get $\overline{E^{\prime}} \cup \overline{E^{\prime \prime}}=Z$ and $\overline{E^{\prime}} \cap \overline{E^{\prime \prime}}=\bar{X}$ from simple calculations, and thus the claim follows from Proposition 3.3.

Corollary 3.7. The modular join of two matroids in $\mathscr{M}$ is again in $\mathscr{M}$.

Proof. Let $M^{\prime}, M^{\prime \prime} \in \mathscr{M}$ have a modular flat $X=E^{\prime} \cap E^{\prime \prime}$ in common. Then the modular join $P=P_{X}\left(M^{\prime}, M^{\prime \prime}\right)$ is again binary by Proposition 3.2(1). However, since the Fano plane is not a modular join, every Fano plane $F \subseteq E$ in $P$ is by Proposition 3.6 (putting $Y=\widehat{0}$ and $Z=F$ ) contained in $E^{\prime}$ or $E^{\prime \prime}$.

Lemma 3.8 [5, Theorem 7.5]. Let $P=P_{X}\left(M^{\prime}, M^{\prime \prime}\right)$ be a modular join. Then

$$
\chi_{P}(t)=\chi_{M^{\prime}}(t) \cdot \chi_{M^{\prime \prime}}(t) / \chi_{X}(t) .
$$

In particular, if $\chi_{M^{\prime}}(t)$ and $\chi_{M^{\prime \prime}}(t)$ both factor into linear factors in $\mathbb{Z}[t]$, then so does $\chi_{P}(t)$.

Proof. This is computed in [5], reducing to Stanley's Modular Factorization Theorem [16] via a study of the complete Brown truncation. A nicer proof results from the observation that if $\omega$ is a linear ordering on $E=E^{\prime} \cup E^{\prime \prime}$ in which $X$ is an initial segment, then the corresponding broken circuit complex $\Delta=\mathrm{BC}(P, \omega)$ factors as $\Delta=\left.\left.\left.\Delta\right|_{X} * \Delta\right|_{E^{\prime}-X} * \Delta\right|_{E^{\prime \prime}-X}$, where $\left.\left.\Delta\right|_{X} * \Delta\right|_{E^{\prime}-X}=$ $\left.\Delta\right|_{E}=\mathrm{BC}\left(M,\left.\omega\right|_{E^{\prime}}\right)$ and $\left.\left.\Delta\right|_{X} * \Delta\right|_{E^{\prime \prime}-X}=\left.\Delta\right|_{E}=\mathrm{BC}\left(M,\left.\omega\right|_{E^{\prime \prime}}\right)$ - with the Modular Shortcut Axiom [5, Theorem 3.11] this easily follows from the definitions. Consult $[6,3]$ for this approach.

Theorem 3.9. Let $\mathscr{S}$ be a class of supersolvable geometric lattices that is closed under taking intervals, and let $\mathscr{S}^{*}$ be the smallest class of geometric lattices containing $\mathscr{S}$ that is closed under taking modular joins. Then

(i) every interval of a lattice in $\mathscr{S}^{*}$ is a lattice in $\mathscr{S}^{*}$, and 
(ii) the characteristic polynomial of any lattice in $\mathscr{S}^{*}$ factors over $\mathbb{Z}$ into linear factors.

The extra generality allowing for subclasses of supersolvable geometric lattices in Theorem 3.9 applies, in particular, to the case where $\mathscr{S}$ is

(1) the class of all finite supersolvable geometric lattices,

(2) the class of all binary supersolvable geometric lattices,

(3) the class of all unimodular supersolvable geometric lattices,

(4) the class of all graphic supersolvable geometric lattices.

In cases (3) and (4) (which coincide by Theorem 2.7) we see $\mathscr{S}^{*}=\mathscr{S}$, whereas in cases (1) and (2) this does not hold.

At this point, we also observe that a practical property of supersolvable matroids, namely, that they allow convenient proofs by induction on the size of the ground set, does not get lost under forming modular joins.

Lemma 3.10. Let $X$ be a minimal flat of $P$ such that $P$ is a modular join $P=P_{X}\left(M^{\prime}, M^{\prime \prime}\right)$ over $X$. Assume $X \neq E^{\prime}$, where $M^{\prime}=P \mid E^{\prime}$ is supersolvable. Then there is an $e \in E^{\prime}-X$ such that $P_{X}\left(M^{\prime}, M^{\prime \prime}\right)-e=P_{X}\left(M^{\prime}-e, M^{\prime \prime}\right)$ and $M^{\prime}-e$ is again supersolvable.

Proof. Let $H^{\prime}$ be a modular hyperplane of $M^{\prime}$. Minimality of $X$ implies (as in the proof of Theorem 3.4) that $X \subseteq H^{\prime}$. With this $e$ can be chosen arbitrarily in $E^{\prime}-H^{\prime}$.

\section{MOdULAR FLATS IN BINARY MATROIDS}

The possible structures of modular flats in binary matroids are quite restricted. This was already indicated by Theorem 2.7 , and will reappear in Theorem 4.7 below. We first collect a few elementary facts. One observation is that modular flats require the existence of many 3-circuits (3-point lines). In fact supersolvable matroids are characterized by their families of 3-circuits in [3].

Lemma 4.1 [5, Corollary 3.15]. Let $M$ be a simple connected binary matroid. $A$ line $X$ in $M$ is modular if and only if it has 3 points (that is, if it is a 3-circuit). Proof. Proposition 2.3(4) and 2.3(3).

From Proposition 2.2(4) we indeed get that every complete flat (flat $X$ with $2^{r(X)}-1$ points) in a binary geometry is modular. Thus in particular, every Fano plane in a simple binary matroid is modular, so that Theorem 2.7 can be interpreted as a statement about modular flats in binary geometries.

We now start to collect existence theorems for modular flats in binary matroids (that fail in general matroids).

Lemma 4.2. Let $M$ be a binary supersolvable matroid, $r(M) \geq 2$. Then every point in $M$ is contained in a modular line.

Proof. Assume $M$ is simple and with Lemma 4.1 that some $p \in E$ does not lie on a 3-point line. Let $H$ be a modular hyperplane. By induction on the 
rank and Proposition 2.2(1a) we may assume that $p \notin H$. Then there cannot be another $p^{\prime} \in E-H$, because modularity of $H$ would require a third point on the line $\overline{\left\{p, p^{\prime}\right\}}$. Thus $p$ is the only point of $M$ not on $H$, and thus a coloop (distributive), and every line through $p$ is modular.

However, this cannot be continued to higher ranks in binary matroids.

Example 4.3. Let $M$ be the projective space of rank 4 over $\mathrm{GF}(2)$, from which a basis has been deleted. For example, we can represent this as

$$
M=\mathrm{PG}(3,2)-\{0001,0011,0101,1001\} .
$$

This binary matroid of rank 4 is supersolvable, because the hyperplane $\left\{x_{4}=0\right\}$ is a complete Fano plane-this is the only Fano plane in $M$. However, $M$ also contains the modular line $X=\{1000,1111,0111\}$, which is not contained in any modular hyperplane; this is most easily seen from the characteristic polynomial $\chi(t)=(t-1)(t-2)(t-4)^{2}$, which by Theorem 2.5(1) implies that every modular hyperplane of $M$ has to be a Fano plane. In particular the (modular) line $X$ and the (modular) points 1111 and 0111 on $X$ are not contained in an $M$-chain. Thus, by Theorem 3.4 , if $M^{\prime}$ is an isomorphic copy of $M$ with $E \cap E^{\prime}=X$, then $P_{X}\left(M, M^{\prime}\right)$ is not supersolvable.

However, for supersolvable matroids in the class $\mathscr{M}$ we can prove that every modular flat $X<\hat{1}$ is contained in a modular flat of rank $r(X)+1$.

Theorem 2.7 reduces this to the case of graphic matroids, where Proposition $2.3(3)$ reduces to the case of a connected graphic matroid $M(G)$, that is, a 2-connected graph $G$.

Proposition 4.4 ([16], compare to [5, Proposition 4.3.2]). Let $G=(V, E)$ be a 2-connected graph, and $M=M(G)$ its matroid.

(1) $B \subseteq E$ is a modular flat of $M$ if and only if

(i) $B$ is the edge set of a 2-connected induced subgraph $G\left[V_{B}\right]=$ $\left(V_{B}, B\right)$ of $G$.

(ii) The set of neighbors in $V_{B}$ of the vertices of any connected component of $G-V_{B}$ forms a clique in $G\left[V_{B}\right]$.

(2) In particular, $H \subseteq E$ is a modular hyperplane of $M$ if and only if $H$ is the edge set of $G-v$ for some simplicial vertex $v$ of $G$, that is, some vertex $v$ whose neighbors form a clique in $G$.

We note that if $G$ is not 2-connected, then we can assume that $G$ is a disjoint union of its 2-connected blocks, and thus the modular hyperplanes of $M(G)$ again are exactly the complements of simplicial vertices in $G$.

Proposition 2.6 ("chordal graphs have a supersolvable matroid") thus requires the existence of a simplicial vertex in every chordal graph with $E \neq \varnothing$. The classical result, due to Dirac [8], states even more.

Lemma $4.5[8,5]$. Let $G=(V, E)$ be a chordal graph. Then either $G$ is a complete graph $\left(G=K_{n}, n \geq 1\right)$, or $G$ has two nonadjacent simplicial vertices. 
Putting things together, we get:

Theorem 4.6. The class of binary supersolvable matroids without a Fano plane is generated by the matroids $M\left(K_{n}\right) \quad(n \geq 2)$ by the operation of forming the modular join of two matroids of equal rank in a flat that is a modular hyperplane in both. Furthermore, the matroids $M\left(K_{n}\right)$ do not arise as a modular join of smaller matroids for $n \geq 2$.

Proof. Let $M \in \mathscr{M}$ be supersolvable, then $M=M(G)$ for a chordal graph $G$ by Theorem 2.7 and Proposition 2.6. Thus either $M=M\left(K_{n}\right)$ for some $n \geq 1$, or $M$ has two modular hyperplanes $H_{1}$, and $H_{2}$ such that $H_{1} \cup H_{2}=E$, by Lemma 4.5 and Proposition 4.4, where $H_{1}$ and $H_{2}$ arise as the edge sets of $G-v_{1}$ and $G-v_{2}$ for some nonadjacent simplicial vertices $v_{1}$ and $v_{2}$ in $G$. Thus from Proposition 3.3 we get $M=P_{X}\left(M\left(G_{1}\right), M\left(G_{2}\right)\right)$ for $X=H_{1} \cap H_{2}$ where $r\left(M\left(G_{1}\right)\right)=r\left(M\left(G_{2}\right)\right)=r(M)-1$.

Observe here that the modular join decomposition is much more general than the "simplicial tree decomposition" of graphs as surveyed in [7]. In fact every chordal graph has a simplicial tree decomposition, but for this the join cannot be required to be formed in hyperplanes.

It is interesting to note that modular joins do not in general preserve supersolvability, although modular joins in hyperplanes do. The reason is that in a supersolvable matroid, not every modular flat is contained in an $M$-chain. Example 4.3 demonstrates a binary example of this. However, every modular hyperplane extends to an $M$-chain, by Theorem 2.5(2) together with Proposition 2.2(1a). In particular, with Theorem 3.4 this means that the operation of forming modular joins in hyperplanes used for Theorem 4.6 does preserve supersolvability.

Again we can prove more for the matroids in the class $\mathscr{M}$. Of course, Theorem 4.7 below is again a result about graphs, respectively graphic matroids, which only by Theorem 2.7 is lifted to the class $\mathscr{M}$.

Theorem 4.7. (i) Let $M$ be a supersolvable matroid in the class $\mathscr{M}$, and let $X$ be a modular flat. Then $X$ is contained in an $M$-chain of $M$.

(ii) The modular join $P_{X}\left(M^{\prime}, M^{\prime \prime}\right)$ of two supersolvable matroids $M^{\prime}, M^{\prime \prime} \in$ $\mathscr{M}$ is again a supersolvable matroid in $\mathscr{M}$.

Proof. For this one considers an isomorphic copy $M^{\prime}$ of $M$ such that $E \cap E^{\prime}=$ $X$, and the modular join $P_{X}\left(M, M^{\prime}\right)$. Corresponding to this there are graphs $G=(V, E)$ and $G^{\prime}=\left(V^{\prime}, E^{\prime}\right)$ such that $G\left[V \cap V^{\prime}\right]=\left(V \cap V^{\prime}, X\right)$, such that $M=M(G), M^{\prime}=M\left(G^{\prime}\right)$, and $P_{X}\left(M, M^{\prime}\right)=M\left(G_{0}\right)$, where $G_{0}=$ $\left(V \cup V^{\prime}, E \cup E^{\prime}\right)$ is the modular join of $G$ and $G^{\prime}$ in $X$.

Now $G$ and $G^{\prime}$ are both chordal. With Proposition 4.4(1) one sees that $G_{0}$ is also chordal, because every cycle that meets both $E^{\prime}-E$ and $E-E^{\prime}$ has a chord in $X$, hence $P_{X}\left(M, M^{\prime}\right)$ is supersolvable, which proves (ii). Thus Theorem 3.4 implies part (i). 
The "doubling" trick in this proof (and in Example 4.3) was also used by Lindström [13, Theorem 1].

\section{ACKNOWLEDGMENT}

The author is grateful to Bob Bixby, Dan Kleitman, and Klaus Truemper for valuable discussions. He thanks Doris Konnerth for moral and secretarial support.

\section{REFERENCES}

1. K. Baclawski and N. White, Higher order independence in matroids, J. London Math. Soc. (2) 19 (1979), 193-202.

2. F. Barahona and M. Grötschel, On the cycle polytope of a binary matroid, J. Combin. Theory Ser. B 40 (1986), 40-62.

3. A. Björner and G. M. Ziegler, Broken circuit complexes: factorizations and generalizations, J. Combin. Theory Ser. B 51 (1991), 96-126.

4. R. C. Bose and R. C. Burton, A characterization of flat spaces in a finite geometry and the uniqueness of the Hamming and the MacDonald codes, J. Combin. Theory 1 (1966), 96-104.

5. T. Brylawski, Modular constructions for combinatorial geometries, Trans. Amer. Math. Soc. 203 (1975), 1-44.

6. __ The broken-circuit complex, Trans. Amer. Math. Soc. 234 (1977), 417-433.

7. R. Diestel, Simplicial decompositions, tree decompositions and graph minors, Proc. Eleventh British Combin. Conf., London, 1987, Ars Combin. 25C (1988), 97-104.

8. G. A. Dirac, On rigid circuit graphs, Abh. Math. Sem. Univ. Hamburg 25 (1961), 71-76.

9. M. C. Golumbic, Algorithmic graph theory and perfect graphs, Academic Press, New York, 1980.

10. M. D. Halsey, Line-closed combinatorial geometries, Discrete Math. 65 (1987), 245-248.

11. I. Heller, On linear systems with integral valued solutions, Pacific J. Math. 7 (1957), 1351 1364.

12. J. P. S. Kung, Growth rates and critical exponents of classes of binary geometries, Trans. Amer. Math. Soc. 293 (1986), 837-859.

13. B. Lindström, On strong joins and pushouts of combinatorial geometries, J. Combin. Theory Ser. A 25 (1978), 77-79.

14. U. S. R. Murty, Extremal matroids with forbidden minors, Proc. Seventh Southeastern Conf. Combinatorics, Graph Theory, and Computing, Baton Rouge, 1976, Congr. Numer. XVII (1976), 463-468.

15. P. D. Seymour, Decomposition of regular matroids, J. Combin. Theory Ser. B 28 (1980), 305-354.

16. R. P. Stanley, Modular elements of geometric lattices, Algebra Universalis 1 (1971), 214217.

17. __ Supersolvable lattices, Algebra Universalis 2 (1972), 197-217. 
18. D. J. A. Welsh, Matroid theory, Academic Press, London, 1976.

19. N. White (ed.), Theory of matroids, Cambridge Univ. Press, Cambridge, 1986.

20. (ed.), Combinatorial geometries, Cambridge Univ. Press, Cambridge, 1987.

InStitut für MAthematik, Universität AUgSBURg, UniversitätsSTrasse 8, D-8900 AUgsBURG, West GeRMANY 